Rapport - Société canadienne d'histoire de l'Église catholique

\title{
Sociologie d'un diocèse canadien : aspects méthodologiques
}

\section{Fernand Dumont}

Volume 24, 1956-1957

URI : https://id.erudit.org/iderudit/1007431ar

DOI : https://doi.org/10.7202/1007431ar

Aller au sommaire du numéro

Éditeur(s)

La Société canadienne d'histoire de l'Église catholique

ISSN

0318-6148 (imprimé)

1927-7075 (numérique)

Découvrir la revue

Citer cet article

Dumont, F. (1956). Sociologie d'un diocèse canadien : aspects méthodologiques. Rapport - Société canadienne d'histoire de l'Église catholique, 24, 31-38.

https://doi.org/10.7202/1007431ar

Tous droits réservés @ La Société canadienne d'histoire de l'Église catholique, 1958
Ce document est protégé par la loi sur le droit d'auteur. L’utilisation des services d'Érudit (y compris la reproduction) est assujettie à sa politique d'utilisation que vous pouvez consulter en ligne.

https://apropos.erudit.org/fr/usagers/politique-dutilisation/ 


\section{Sociologie $d$ 'un diocèse canadien: Aspects méthodologiques}

Le titre de mon exposé vous l'indique déjà : je ne suis pas historien, mais sociologue et je vous parlerai du présent plutôt que du passé de notre société religieuse. Mais les historiens savent mieux que quiconque à quoi s'en tenir sur l'actualité; ils tiennent que le présent n'est, après tout, qu'un découpage opéré sur le passé. Non seulement le sociologue leur donne raison sans peine, mais il emprunte lui-même très souvent les chemins de l'histoire dans son travail de description et d'explication des socićtés humaines.

$\mathrm{Au}$ surplus, la sociologie religieuse est, au Canada français, une très jeune discipline. Dès que le sociologue se penche sur notre société religieuse, comment ne songerait-il pas à nos historiens qui, depuis longtemps déjà, s'attachent à en retracer la genèse ? M. Gabriel Le Bras, un des maîtres contemporains de la sociologie religieuse et lui-même grand historien, a souvent insisté sur la nécessaire collaboration de la sociologie avec l'histoire. "A tous ses fidèles, écrit-il, l'Eglise romaine impose unité de foi et de discipline. Le contenu de l'un et l'autre corpus varie : celui des croyances s'enrichit de définitions dogmatiques; celui des institutions s'adapte aux nécessités changeantes. Rechercher les causes, les caractères, les effets sociaux de ces mutations : tel est l'office du sociologue. Il sait qu'une société vit dans le temps et ne cesse de modifier ses formes ${ }^{1}$. Je voudrais que mon exposé d'aujourd'hui vous apparaisse dans cette perspective. J'aimerais en faire un appel à un dialogue - qui m'apparaît nécessaire - entre nos deux disciplines. La meilleure façon, il me semble, d'en arriver à ce résultat, c'est non pas de vous parler des ambitions de la sociologie en général, mais bien plutôt de vous montrer à l'œuvre notre propre travail d'investigation : quitte à illustrer ensuite par quelques exemples les problèmes qui s'en dégagent pour la recherche historique.

En avril 1956, Son Excellence $M^{g r}$ Emilien Frenette, évêque de Saint-Jérôme, confiait au Centre de Recherche de la Faculté des Sciences sociales de l'Université Laval une analyse sociologique de son diocèse. J'ai mené cette étude avec la collaboration constante de mon collègue et ami, M. Yves Martin, et l'aide d'une équipe d'assistants composée de MM. Robert Sévigny, Marc Lessard, Gérard Lapointe et Vincent Lemieux; mon collègue, M. Napoléon Leblanc, a aussi apporté une précieuse contribution à cette recherche. Une première enquête menée durant l'été de 1956 a donné lieu à un volumineux rapport remis à Monseigneur l'Evêque en mai dernier; une seconde enquête a été

1 Gabriel Le Bras, Etudes de sociologie religieuse, tome II, p. 665. 
poursuivie cet été, mais le dépouillement des matériaux n'est pas encore complété. C'est l'appareil méthodologique de cette étude que je voudrais vous présenter, en laissant de côté les éléments trop techniques et en tâchant de ne pas abuser des concepts un peu barbares qu'utilise la sociologie.

La tâche qui nous était confiée n'avait rien de très nettement circonscrit : on ne nous demandait pas d'étudier tel type de comportement religieux ou tel aspect particulier des structures paroissiales ou des associations religieuses. On voit les dimensions du problème : la vie religieuse dans une région comptant quarante-huit paroisses.

La vie de l'Eglise dans une société donnée se situe évidemment surtout au niveau de l'organisation sociale et des mentalités. En définitive, c'est dans la vie des institutions ecclésiales et surtout dans les attitudes des fidèles que l'on peut sonder les profondeurs de la vitalité religieuse. On se demandera alors pourquoi nous ne sommes pas allés directement aux institutions et aux comportements. C'est que les institutions religieuses ne sont pas les seules à encadrer les adhérents de l'Eglise. Ceux-ci sont insérés dans tout un réseau d'institutions, dont la paroisse et les associations catholiques ne constituent qu'un secteur : il faut d'abord restituer à ce complexe sa figure d'ensemble pour en comprendre un élément quel qu'il soit. Mais surtout on ne peut se contenter d'analyser les comportements religieux des individus, leur pensée et leurs attitudes, sans avoir d'abord éclairé leur situation sociale. Qu'on soit ouvrier ou professionnel, qu'on jouisse de la sécurité de l'emploi ou qu'on soit ballotté d'une occupation à l'autre : tout cela peut fort bien être indifférent à la qualité surnaturelle de notre vie religieuse; cela ne l'est sûrement pas aux démarches par lesquelles tel ou tel individu vit sa foi dans le monde. La situation sociale de l'individu constitue une des déterminations essentielles de ses comportements religieux ou autres.

Mais prenons-y garde : on ne peut se contenter, pour situer un individu, d'obtenir de lui quelques renseignements sur son emploi, son revenu, etc. Pour prendre un exemple extrême : on peut gagner six dollars par jour dans une grande ville et subsister à peine, en gagner six dans telle petite localité et y être à l'aise. La situation sociale de l'individu est relative à celle des autres; elle renvoie à un véritable système de situations complémentaires de la sienne et qui définissent celle-ci. Ce système dépend évidemment lui-même, dans sa configuration générale, comme dans ses aspects particuliers, de l'ensemble de la société en cause : de son économie, des caractères de sa population, etc. La situation de l'individu est définie, au sens strict du terme, par le système social où il est impliqué.

Pour étudier les institutions et les attitudes religieuses, nous sommes donc renvoyés à l'ensemble de cette société que constitue le diocèse. Ce qui n'implique pas évidemment une description exhaustive; il ne s'agissait pas de réunir pêle-mêle un monceau de données géographiques, 
historiques, etc., nous permettant de décrire minutieusement le territoire impliqué. $\mathrm{La}$ théorie sociologique nous incite justement à faire intervenir l'explication dès le départ dans l'analyse des ensembles sociaux : c'est-à-dire qu'elle permet de choisir des indices caractéristiques des divers éléments d'une société, de les sélectionner au niveau qu'imposent les ressources financières, le personnel de chercheurs, la documentation disponible, etc. Ainsi, partir de la " totalité * du social, ce n'est pas juxtaposer des éléments infinis; c'est esquisser, par approches successives, une configuration de plus en plus précise.

Au niveau de l'étude scientifique, on voit alors comment se présente l'analyse des problèmes religieux sur le territoire du diocèse. Les phénomènes religieux apparaissent comme une des « variables 》 de cet ensemble social dont nous venons de parler : comment cette variable religieuse influence l'ensemble social; comment l'ensemble social modèle la vie religieuse; comment celle-ci s'enracine dans l'ensemble social, l'organise, l'aménage et ne le transcende qu'en l'informant... Dans les limites de l'analyse sociologique, la « variable » religieuse s'explique par toutes les autres variables de l'ensemble social et contribue à les expliquer.

Voilà qui définit l'orientation globale de nos recherches : les exigences de la mission diocésaine, la nature même de la réalité à étudier nous obligeaient à reconstituer d'abord la structure sociale du diocèse. Mais le territoire impliqué est vaste et divers : quel rapport peut bien unir, à première vue, Argenteuil et Terrebonne, Montfort et SainteThérèse-de-Blainville? Tout en visant essentiellement les ensembles, l'analyse structurelle ne doit pas en masquer la diversité. D'où une démarche méthodologique qui s'imposait: découper le territoire du diocèse en zones humaines plus restreintes. Ce qui permettait d'assumer, au départ, la diversité des localités, des « microcultures»; et ce qui constituait du même coup une première étape de généralisation pour parvenir à la structure globale.

Le découpage de ces zones n'a pas été fait au hasard. Il a été opéré par étapes, par tentatives successives au cours du travail sur le terrain. Remarquons seulement que, dès le départ, le premier critère a été sociologique. Il référait à la façon même dont des gens de la société impliquée en voyaient le fractionnement : au cours d'interviews préliminaires, nous avons demandé à certaines personnes choisies en raison de leur "situation 》 de partager le diocèse en petites régions. Leurs réponses, transcrites sur cartes, ont fourni le point de départ de nos sectionnements successifs du territoire. Tenant compte d'un certain nombre de compromis techniques inévitables dans des recherches de ce genre et dont il n'y a pas lieu de parler ici, nous nous sommes arrêtés à une division du territoire en dix zones.

Il s'agissait donc de considérer, en première approximation, chacune de nos zones comme une structure sociale restreinte. Le problème 
crucial de la recherche se posait alors : répartir cet ensemble en variables qui soient définies à un niveau accessible aux moyens d'investigation dont nous disposions. Or ces moyens étaient limités. Les recensements du Canada fournissent des données statistiques abondantes, mais ils comportent souvent, pour un travail comme celui-ci, de graves lacunes. Les inventaires des ressources naturelles et industrielles des comtés, élaborés par l'Office économique provincial, nous ont été communiqués : ils comportent des renseignements précieux que nous avons largement utilisés, mais si l'inventaire des Deux-Montagnes date de 1955, celui d'Argenteuil remonte à $194 \mathrm{l}$ : beaucoup de ces données ont donc vieilli et étaient proprement inutilisables; l'Office a fait, pour certaines municipalités seulement, une remise à jour de quelques chiffres. La plupart du temps, les renseignements fournis par les inventaires ne sont que du matériel brut devant être traité par l'analyse sociologique; de plus ces documents ne comportent que de très rares indications sur l'organisation sociale et la culture. Les rapports de messieurs les curés à Monseigneur l'Evêque nous ont fourni aussi des données statistiques abondantes; on sait par ailleurs que leur valeur n'est pas toujours très sûre. Si l'on ajoute l'ouvrage de Raoul Blanchard (souvent superficiel) et quelques monographies historiques, l'on aura à peu près toute la documentation disponible. Tout cela est très mince si l'on compare à d'autres régions de la province pour lesquelles existent au moins quelques études économiques ou géographiques. C'est en tenant compte de ces lacunes des documents que nous avons élaboré nos interviews dans la première phase de la recherche. Nous n'avons alors visé qu'incidemment à détecter des attitudes; avant tout il s'est agi d's interviews d'information" visant à repérer les indices de structure sociale que ne pouvait fournir l'analyse des documents.

Pour chacune des zones, nous avons isolé les grandes variables suivantes : la population, la structure économique, les occupations, l'organisation sociale, la culture. Ce ne sont pas là, remarquons-le à nouveau, de simples juxtapositions d'éléments disparates : chacune de ces variables renvoie fonctionnellement aux autres, explique celles-ci et est expliquée par elles. Ainsi la structure économique renvoie évidemment à l'analyse de la population (main-d'œuvre disponible, etc.) et explique la structure de l'emploi et des occupations; l'organisation sociale est liée à la structure économique (présence ou absence de syndicats, types de mouvements d'Action catholique spécialisée, etc.).

Etant donné les précisions fournies plus haut, on comprendra que nous ayons consacré toute la première phase de notre recherche à cette analyse de la société en général sans nous arrêter particulièrement aux institutions religieuses. Mais insistons aussi sur ce point : l'importance accordée à ce premier aspect du travail n'en est pas seulement une d'antériorité méthodologique. Sous un certain aspect, toute analyse sociologique est à portée immédiatement religieuse puisque, du point de vue de la foi, rien de la situation de l'homme n'est indifférent à l'Eglise et au salut. Comme l'écrit un théologien : * Si le progrès humain n'est pas comme tel efficace de salut, il constitue du moins ce qui est sauvé, 
selon le mot d'Augustin sur la liberté... Chacun de nous sera éternellement ce qu'il se sera fait sur la terre. Et de même les nouveaux cieux et la nouvelle terre seront-ils la transfiguration de ce monde tel que le travail de l'homme aura contribué à la constituer. En ce sens l'histoire des civilisations, comme celle du cosmos, rentre-t-elle dans la vision totale de l'histoire du salut ${ }^{2}$. " Donc, du point de vue du pasteur, toute analyse d'une réalité sociale quelconque est déjà sociologie religieuse puisque le travail de civilisation n'est pas indifférent à l'œuvre de mission. Et précisément la perspective méthodologique adoptée dans notre recherche et qui n'a été définie que du seul point de vue de l'analyse scientifique n'est pas sans analogies avec cette perspective du pasteur.

Au premier stade de notre analyse, les phénomènes religieux apparaissent comme une variable parmi d'autres de la structure sociale.

Dans la seconde phase - celle où nous considérons ces phénomènes religieux pour eux-mêmes - ils se présentent au strict point de vue de la science sociologique, comme une variable d'une nature toute particulière : au Canada français particulièrement, la religion constitue un des éléments les plus importants de la superstructure de la société, un des réseaux culturels principaux où la société se définit elle-même. L'Eglise diocésaine est, de soi, une tentative d'aménagement de l'ensemble de la structure sociale dont nous avons tenté de retracer la figure dans la première étape de notre travail.

L'Eglise diocésaine est un aménagement de la structure sociale en deux sens étroitement complémentaires : elle organise le milieu, elle le définit.

L'Eglise s'inscrit d'abord dans cette variable que nous avons appelée « l'organisation sociale». Tout le territoire est partagé en paroisses; des associations diverses se proposent de rejoindre les individus dans leur situation sociale même (L.0.C., L.I.C.F., etc.). D'autre part, l'Eglise s'insère aussi au plan de la culture : elle aussi a sa mentalité où se reflète le milieu; les pasteurs, les «leaders » des diverses associations chrétiennes ont des objectifs d'action qui se réfèrent évidemment, d'une part, à une doctrine surnaturelle mais aussi, d'autre part, à une connaissance, à une conception du milieu social où doivent s'élaborer conquêtes et stratégies.

Dès lors le problème central, l'interrogation sous-jacente à toute la recherche se précisent : les organisations religieuses (paroisses, associations) assument-elles effectivement la réalité sociale? Les objectifs des pasteurs et des * leaders * laïques sont-ils liés organiquement avec les problèmes posés par la configuration de la structure sociale globale? Ce ne sont pas là évidemment les seules questions qui doivent préoccuper les pasteurs; mais ce sont celles auxquelles la sociologie, avec les ressources de ses concepts et de ses techniques, peut tâcher de répondre.

2 Jean Daniélou, Essai sur le mystère de l'histoire, Ed. du Seuil, p. 22. 
Divers indices peuvent être utilisés pour étudier le problème ainsi posé. Si l'on compare la structure des occupations telle que nous avons essayé de l'éclairer dans la première partie de notre recherche avec une classification des occupations des membres des diverses associations, nous aurons des indications sur la correspondance des situations sociales des « leaders" religieux avec celles que révèle la structure sociale. Par exemple, la Ligue du Sacré-Cour se définit comme un a mouvement d'Action catholique générale : cela suppose que ses membres n'appartiennent pas à un seul ou à quelques secteurs de la structure des occupations... Etant donné l'arrière-plan que constitue la première partie de la recherche et la valeur privilégiée qui y a été donnée à l'indice occupation pour la détection des situations sociales, la vérification devient facile à opérer.

Ce n'est là qu'un exemple. Il a fallu aller plus loin et, par des interviews suffisamment abondantes, détecter chez les a leaders " religieux leur vision du milieu, leurs objectifs et, plus profondément encore, leur propre enracinement dans la société considérée. Nous arrivons ainsi au problème central : quels sont, dans une société comme celle que nous avons étudiée, les mécanismes sociaux de sélection des élites religieuses? En d'autres termes : comment et pourquoi devient-on membres et dirigeants d'associations religieuses dans le diocèse de Saint-Jérôme?

Nous pourrons savoir ainsi si l' « organisation " et la " définition " du milieu social par l'Eglise - dont nous avons parlé plus haut coïncident avec les traits essentiels de la structure sociale ou si, au contraire, elles sont l'œuvre de groupes restreints (une classe sociale par exemple) ou même d'un groupe marginal. Problème capital, on le voit. Une autre question lui est liée, qui n'en est qu'un corollaire, mais qui revêt une importance très grande : jusqu'à quel point les organisations religieuses ne sont-elles qu'un médium psychologique d'intégration sociale pour des gens incapables de retrouver, par d'autres moyens, un certain statut de prestige?

\section{III}

J'ai déjà dit que le premier rapport soumis à Son Excellence Monseigneur l'Evêque de Saint-Jérôme était considérable; le second qui portera sur les institutions et les attitudes religieuses ne le sera pas moins. Il serait donc vain de vouloir donner ici, en quelques pages, un résumé des résultats complexes de nos recherches. Je voudrais plutôt tenter d'indiquer la nature des problèmes, des suggestions que pareille investigation peut fournir à l'historien des sociétés religieuses.

A première vue, il y a peu de chose à espérer de ce côté. Le diocèse de Saint-Jérôme est bien jeune; il n'existe que depuis six ans. Mais il serait éminemment superficiel de s'arrêter à une constatation de ce genre. Replaçons-nous plutôt devant l'attitude fondamentale qui est sous-jacente à la méthode de l'historien. Des travaux bien connus sur la méthodologie historique (ceux de Weber, Aron, Marroux, pour ne 
mentionner que les plus célèbres) ont montré comment l'historien interroge le passé à la lumière du présent. On peut même ajouter que souvent ce sont de grossiers jugements de valeurs ou des préjugés incohérents qui fournissent ce bagage préliminaire plus ou moins assumé par la conscience claire de l'historien et dont le poids est si lourd sur son travail ultérieur de critique et de synthèse. On est ainsi conduit à ce curieux paradoxe : si « l'histoire se fait avec des documents » selon une formule lapidaire du célèbre traité de Langlois et Seignabos, elle se fait aussi avec la conscience qu'a l'historien du présent de la société dont il se propose de retracer le passé.

C'est sur ce plan qu'apparaît, dans toute sa profondeur, l'utilité de la sociologie religieuse pour l'historien de la religion. Elle lui fournit une vision nuancée, diversifiée des problèmes qui se posent actuellement à la société religieuse; elle le munit du même coup d'un bagage de questions et de problèmes bien supérieur - on l'admettra sans peine à ce que pourrait lui suggérer le sens commun. L'analyse du diocèse de Saint-Jérôme révèle, comme dans un microcosme, tout le milieu canadien-français : les structures sociales des «zones» dont j'ai parlé se retrouvent sur toute la carte de la province de Québec avec les problèmes religieux qui en résultent : régions bloquées dans leur développement économique et social; territoires ruraux prospères où perce déjà, sinon la réalité, du moins la nécessité de transformations; zones rurales en mutations où les occupations urbaines exercent sur les jeunes un irrésistible attrait; centres industriels enfin. Partout la mentalité religieuse et les objectifs de la pastorale sont confrontés à des situations et à des problèmes qui n'apparaissent nouveaux qu'à des regards superficiels. Comment une historiographie religieuse soucieuse de ne pas se limiter aux grands hommes ou à la hiérarchie ne puiseraitelle pas là des questions cruciales qu'il faudrait poser à notre passé ?

On a souventes fois souligné, par exemple, la pauvreté de beaucoup d'histoires de paroisse. Et pourtant la nécessité de ce type de monographie est incontestable si nous voulons connaître la vie du peuple et des groupes. L'analyse de la conjoncture qu'affrontent actuellement nos paroisses est bien de nature à renouveler ce genre historique. Après avoir constaté et analysé la crise actuelle des vocations dans telle paroisse rurale, la fortune diverse des œuvres, la crise de conscience de sa population acculée à de nouveaux genres de vie, l'historien de la paroisse s'inquiétera de bien autre chóse que la liste des curés ou des marguilliers.

Une des constatations les plus intéressantes à laquelle nous a mené notre étude du diocèse de Saint-Jérôme, c'est que la mentalité de beaucoup de leaders de mouvements d'Action catholique n'est pas accordée à la nouveauté, à l'originalité de ces institutions. On discerne chez plusieurs ce que nous appellerons « l'esprit de confrérie ». Ici encore, je ne résumerai pas les analyses de détail que nous avons poursuivies. Mais cette mentalité dont nos techniques permettent l'investigation, le sociologue sent tout de suite qu'elle a des racines historiques 
complexes. Quels problèmes passionnants sont alors proposés à l'histoire des confréries encore si peu cultivée chez nous !

Mais je voudrais vous citer un exemple plus probant encore. Dans notre étude, nous avons tâché d'analyser les fondements sociologiques de la paroisse. Personne n'ignore qu'avec les changements sociaux apportés par l'industrialisation et l'urbanisation de notre milieu, la paroisse ne repose plus sur cette homogénéité des relations sociales qui caractérise la société rurale. Ces tensions introduites par des genres de vie nouveaux, générateurs de groupes divers et de classes sociales, rendent évidemment très difficile la communauté paroissiale. Rien n'est plus frappant à cet égard que l'attitude des professionnels et des hommes d'affaires : ils réclament des retraites, des récollections, des sermons pour eux; ils s'imaginent facilement que les difficultés qu'ils éprouvent dans la paroisse urbaine sont propres à leur milieu qu'ils qualifient volontiers de a cultivé ». On voit ainsi apparaître un problème crucial : la division en classes sociales, avec la conscience de groupe qui lui est corrélative et qu'a provoquée l'urbanisation n'est-elle pas un facteur puissant de dissociation de la communauté paroissiale et de la société religieuse elle-même? Il nous semble que l'historien a tout intérêt à projeter ce problème sur notre passé. Il y a longtemps que l'on a commencé à étudier la mentalité religieuse de notre bourgeoisie du XIX ${ }^{\bullet}$ siècle : il suffira de citer les belles études de M. le chanoine Groulx. N'a-t-on pas trop tendance cependant à expliquer la crise religieuse de cette classe sociale au XIX ${ }^{\circ}$ siècle par l'influence néfaste de Voltaire ou d'autres écrivains anti-religieux ? cette crise n'a-t-elle pas aussi ses sources dans la conscience sociale spécifique d'un groupe dans une société déterminée avec ses inévitables répercussions sur la mentalité religieuse ? Il nous paraît certain que pareilles questions pourraient renouveler la problématique traditionnelle de notre historiographie religieuse.

Fernand Dumont. 